\title{
On Commuting Graphs for Elements of Order 3 in Symmetric Groups
}

\author{
Athirah Nawawi and Peter Rowley \\ School of Mathematics \\ University of Manchester \\ Manchester, M13 6PL, U.K. \\ peter.j.rowley@manchester.ac.uk
}

Submitted: May 19, 2012; Accepted: Jan 20, 2015; Published: Feb 9, 2015

Mathematics Subject Classifications: 05C25

\begin{abstract}
The commuting graph $\mathcal{C}(G, X)$, where $G$ is a group and $X$ is a subset of $G$, is the graph with vertex set $X$ and distinct vertices being joined by an edge whenever they commute. Here the diameter of $\mathcal{C}(G, X)$ is studied when $G$ is a symmetric group and $X$ a conjugacy class of elements of order 3 .
\end{abstract}

Keywords: Commuting graph, Symmetric group, Order 3 elements, Diameter

\section{Introduction}

Suppose that $G$ is a finite group and $X$ is a subset of $G$. The commuting graph $\mathcal{C}(G, X)$ is the graph with $X$ as the vertex set and two distinct elements of $X$ being joined by an edge if they are commuting elements of $G$. This type of graph has been studied for a wide variety of groups $G$ and selection of subsets of $G$. One of the earliest investigations occurred in Brauer and Fowler $[8]$ in which $X=G \backslash\{1\}$. This particular case has recently been the subject of further study by Segev [14], [15] and Segev and Seitz [16]. A great deal of attention has been focussed on the case when $X$ is a conjugacy class of involutions - the so-called commuting involution graphs. Pioneering work on such graphs appeared in Fischer [13] which led to the construction of the three Fischer groups. Recently various properties of other commuting involution graphs have been studied; see, for example, [2], $[3],[4],[5],[11]$ and [12]. When $X$ is a conjugacy class of non-involutions, $\mathcal{C}(G, X)$ has to date received less attention. Never-the-less graphs of this type can be of interest - witness the computer-free uniqueness proof of the Lyon's simple group by Aschbacher and Segev [1] which employed a commuting graph whose vertex set consisted of the 3-central subgroups of order 3. Also see Baumeister and Stein [7], the results obtained there being used to describe the structure of Bruck loops and Bol loops of exponent 2. Further, 
commuting graphs when $G$ is a symmetric group have been investigated in Bates, Bundy, Perkins and Rowley [6] and Bundy[9]. The former paper concentrates on the structure of discs (around some fixed vertex) and the diameter of the graph while the latter gives a complete answer as to when $\mathcal{C}(G, X)$ is a connected graph.

In the present paper we shall determine the diameters of $\mathcal{C}(G, X)$ when $G$ is a symmetric group and $X$ is a $G$-conjugacy class of elements of order 3 . So for the rest of this paper we assume $G=\operatorname{Sym}(\Omega)=\operatorname{Sym}(n)$ with $G$ acting upon the set $\Omega=\{1, \ldots, n\}$ in the usual manner. Also let

$$
t=(1,2,3)(4,5,6)(7,8,9) \ldots(3 r-2,3 r-1,3 r) .
$$

Thus $t$ has order 3 and cycle type $1^{n-3 r} 3^{r}$. Set $X=t^{G}$, the $G$-conjugacy class of $t$, and let Diam $(\mathcal{C}(G, X))$ denote the diameter of the commuting graph $\mathcal{C}(G, X)$. Our main results are as follows.

Theorem 1.1. If $n \geqslant 8 r$, then $\operatorname{Diam}(\mathcal{C}(G, X))=2$.

Theorem 1.2. If $6 r<n<8 r$, then $\operatorname{Diam}(\mathcal{C}(G, X))=3$.

Our last theorem only gives a bound on Diam $(\mathcal{C}(G, X))$.

Theorem 1.3. If $r>1$ and $n=6 r$, then Diam $(\mathcal{C}(G, X)) \leqslant 4$.

Consulting Table 1 (or Table 1 of [6]) we see that for $r=1, n=7$ or $r=2, n=15$ we have that Diam $(\mathcal{C}(G, X))=3$ and so Theorem 1.1 is sharp. For $r=2$ the same table gives Diam $(\mathcal{C}(G, X))=4$ when $n=12$ and 2 when $n=16$, so Theorems 1.2 and 1.3 are also sharp. We note that for $r=1$ and $n=6, \mathcal{C}(G, X)$ is disconnected which explains the assumption $r>1$ in Theorem 1.3. All the graphs we consider here are connected - see [9]. For $g \in G, \operatorname{supp}(g)$ denotes the set of points of $\Omega$ not fixed by $g$. We use $d($,$) for the$ usual distance metric on the graph $\mathcal{C}(G, X)$. For $x \in X$, the $i^{\text {th }}$ disc, $\Delta_{i}(x)$, is defined as follows

$$
\Delta_{i}(x)=\{y \mid y \in X \text { and } d(x, y)=i\} .
$$

The proofs of Theorems 1.1, 1.2 and 1.3 adopt a similar, somewhat direct, approach. Since $G$ acting by conjugation upon $X$ induces graph automorphisms on $\mathcal{C}(G, X)$ and of course is transitive on $X$, it suffices to determine (or bound) $d(t, x)$ for an arbitrary vertex $x$ of $X$. This we do by writing down explicit paths in $\mathcal{C}(G, X)$.

\section{Diameter of $\mathcal{C}(G, X)$}

We begin by establishing Theorem 1.1.

\section{Proof of Theorem 1.1}

Let $x \in X$. Set $\Lambda=\operatorname{supp}(t) \cup \operatorname{supp}(x)$ and $s=|\operatorname{supp}(t) \cap \operatorname{supp}(x)|$. Then $|\Lambda|=6 r-s$. If 
$s \geqslant r$, then $|\Lambda| \leqslant 5 r$. Hence there exists $y \in X$ with $\operatorname{supp}(t) \cap \operatorname{supp}(y)=\emptyset=\operatorname{supp}(x) \cap$ $\operatorname{supp}(y)$ and so $d(t, x) \leqslant 2$. Now consider the case $s<r$, and set $e=r-s$. Without loss of generality we may suppose that $\operatorname{supp}(t) \cap \operatorname{supp}(x) \subseteq\{1,2,3, \ldots, 3 s-2,3 s-1,3 s\}$. Put $y_{1}=(3 s+1,3 s+2,3 s+3) \ldots(3 r-2,3 r-1,3 r)$ (so $y_{1}$ is the product of the "last" $r-s=e$ 3-cycles of $t)$. Since $|\Omega \backslash \Lambda|=8 r-(6 r-s)=2 r+s>3 s$ and $s<r$, we may select $y_{2}$ with $\operatorname{supp}\left(y_{2}\right) \subseteq \Omega \backslash \Lambda$ and $y_{2}$ is a product of $s$ pairwise disjoint 3-cycles. So $y=y_{1} y_{2} \in X, t y=y t$ and $x y=y x$. Thus $d(t, x) \leqslant 2$. Clearly Diam $(\mathcal{C}(G, X)) \geqslant 2$, and so the theorem follows.

Before proving Theorems 1.2 and 1.3 we introduce some notation and certain permutations of $\operatorname{Sym}(\Omega)$. These permutations, though elements of order 3, are not in general in $X$. We will assume that $|\Omega| \geqslant 6 r$. For $x \in X$, we let $\left\{\vartheta_{i}(x)\right\}_{i=1, \ldots, r}$ denote the orbits of $\langle x\rangle$ on $\Omega$ of size 3. So $\operatorname{supp}(x)=\bigcup_{i=1}^{r} \vartheta_{i}(x)$. Write $t=t_{1} t_{2} \ldots t_{r}$ where $t_{i}=(3 i-2,3 i-1,3 i)$. So $\vartheta\left(t_{i}\right)=\vartheta_{i}(t)=\{3 i-2,3 i-1,3 i\}$.

Let $x \in X$. Denote the product of the $t_{i}$ 's for which $\vartheta_{i}(t) \cap \operatorname{supp}(x)=\emptyset$ by $\tau_{0}$ and let $\tau_{3}$ be the product of the $t_{i}$ 's for which $\vartheta_{i}(t) \subseteq \operatorname{supp}(x)$. Also let $\tau_{1}$ be the product of $r_{1} t_{i}$ 's where $\left|\vartheta_{i}(t) \cap \operatorname{supp}(x)\right|=1,3 \mid r_{1}$ and $r_{1}$ is as large as possible. Analogously, $\tau_{2}$ is the product of $r_{2} t_{i}$ 's where $\left|\vartheta_{i}(t) \cap \operatorname{supp}(x)\right|=2,3 \mid r_{2}$ and $r_{2}$ is as large as possible. Setting $\tau_{*}=t \tau_{0}^{-1} \tau_{1}^{-1} \tau_{2}^{-1} \tau_{3}^{-1}$ we have $t=\tau_{*} \tau_{0} \tau_{1} \tau_{2} \tau_{3}$. Let $r_{*}$ be the number of $t_{i}$ 's in $\tau_{*}, r_{0}$ the number of $t_{i}$ 's in $\tau_{0}$ and $r_{3}$ the number of $t_{i}$ 's in $\tau_{3}$. Observe that the maximality of $r_{1}$ and $r_{2}$ means $r_{*} \leqslant 4$ and that at most two of the $t_{i}$ 's in $\tau_{*}$ will have $\left|\vartheta_{i}(t) \cap \operatorname{supp}(x)\right|=1$ and at most two will have $\left|\vartheta_{i}(t) \cap \operatorname{supp}(x)\right|=2$. Evidently $r=r_{*}+r_{0}+r_{1}+r_{2}+r_{3}$ and, for $i=0,1,2,3,\left|\operatorname{supp}(x) \cap \operatorname{supp}\left(\tau_{i}\right)\right|=i r_{i}$. Putting $s_{*}=\left|\operatorname{supp}(x) \cap \operatorname{supp}\left(\tau_{*}\right)\right|$, we also have

$$
|\operatorname{supp}(t) \cap \operatorname{supp}(x)|=s_{*}+r_{1}+2 r_{2}+3 r_{3} .
$$

Set $\Lambda=\Omega \backslash(\operatorname{supp}(t) \cup \operatorname{supp}(x))$. Since

$$
\begin{aligned}
|\operatorname{supp}(t) \cup \operatorname{supp}(x)| & =3 r+3 r-\left(s_{*}+r_{1}+2 r_{2}+3 r_{3}\right) \\
& =6 r-\left(s_{*}+r_{1}+2 r_{2}+3 r_{3}\right)
\end{aligned}
$$

it follows that

$$
\begin{aligned}
& |\Lambda|=s_{*}+r_{1}+2 r_{2}+3 r_{3} \text { if } n=6 r \text { and } \\
& |\Lambda| \geqslant 1+s_{*}+r_{1}+2 r_{2}+3 r_{3} \text { if } n>6 r .
\end{aligned}
$$

Since 3 divides $r_{1}$, we may write

$$
\tau_{1}=\prod \mu_{i_{1} i_{2} i_{3}}
$$

where the product of the $\mu_{i_{1} i_{2} i_{3}}=t_{i_{1}} t_{i_{2}} t_{i_{3}}$ is pairwise disjoint. For each $\mu_{i_{1} i_{2} i_{3}}=t_{i_{1}} t_{i_{2}} t_{i_{3}}=$ $\left(3 i_{1}-2,3 i_{1}-1,3 i_{1}\right)\left(3 i_{2}-2,3 i_{2}-1,3 i_{2}\right)\left(3 i_{3}-2,3 i_{3}-1,3 i_{3}\right)$ we may without loss, suppose that $\operatorname{supp}\left(\mu_{i_{1} i_{2} i_{3}}\right) \cap \operatorname{supp}(x)=\left\{3 i_{1}-2,3 i_{2}-2,3 i_{3}-2\right\}$. Put

$$
\lambda_{i_{1} i_{2} i_{3}}=\left(3 i_{1}-2,3 i_{2}-2,3 i_{3}-2\right)\left(3 i_{1}-1,3 i_{2}-1,3 i_{3}-1\right)\left(3 i_{1}, 3 i_{2}, 3 i_{3}\right) .
$$


Then $\lambda_{i_{1} i_{2} i_{3}}$ commutes with $\mu_{i_{1} i_{2} i_{3}}$. Let

$$
\rho_{1}=\prod \lambda_{i_{1} i_{2} i_{3}}
$$

and observe that $\rho_{1}$ commutes with $t$ and will be a pairwise disjoint product of $r_{1} 3$-cycles. Further, $\frac{r_{1}}{3}$ of the 3 -cycles in $\rho_{1}$ will have their support contained in $\operatorname{supp}(x)$ while the remaining $\frac{2 r_{1}}{3} 3$-cycles in $\rho_{1}$ will have their support intersecting $\operatorname{supp}(x)$ in the empty set. Also, as 3 divides $r_{2}$, we may express

$$
\tau_{2}=\prod \eta_{j_{1} j_{2} j_{3}}
$$

where $\eta_{j_{1} j_{2} j_{3}}=t_{j_{1}} t_{j_{2}} t_{j_{3}}$ with the product being pairwise disjoint. For each $\eta_{j_{1} j_{2} j_{3}}$ we may suppose that $\operatorname{supp}\left(\eta_{j_{1} j_{2} j_{3}}\right) \cap \operatorname{supp}(x)=\left\{3 j_{1}-2,3 j_{1}-1,3 j_{2}-2,3 j_{2}-1,3 j_{3}-2,3 j_{3}-1\right\}$. Define

$$
\delta_{j_{1} j_{2} j_{3}}=\left(3 j_{1}, 3 j_{2}, 3 j_{3}\right)\left(3 j_{1}-2,3 j_{2}-2,3 j_{3}-2\right)\left(3 j_{1}-1,3 j_{2}-1,3 j_{3}-1\right),
$$

and let

$$
\rho_{2}=\prod \delta_{j_{1} j_{2} j_{3}}
$$

Evidently $\rho_{2}$ commutes with $t$ and $\rho_{2}$ is a pairwise disjoint product of $r_{2} 3$-cycles. Moreover, $\frac{2 r_{2}}{3}$ of the 3 -cycles in $\rho_{2}$ will have their support contained in $\operatorname{supp}(x)$ and the remaining $\frac{r_{2}}{3}$ have supports intersecting $\operatorname{supp}(x)$ in the empty set.

Let $\sigma_{1}$ (respectively $\sigma_{2}$ ) be the product of the $\frac{2 r_{1}}{3}$ (respectively $\frac{r_{2}}{3}$ ) 3-cycles in $\rho_{1}$ (respectively $\rho_{2}$ ) whose support intersects $\operatorname{supp}(x)$ in the empty set. Also let $\sigma_{4}$ be a pairwise disjoint product of $\left(\frac{r_{1}}{3}+\frac{2 r_{2}}{3}+r_{3}\right) 3$-cycles with $\operatorname{supp}\left(\sigma_{4}\right) \subseteq \Lambda$. Put $\Delta=\Lambda \backslash \operatorname{supp}\left(\sigma_{4}\right)$.

We now summarize the pertinent properties of the permutations just introduced.

Lemma 2.1. (i) $\operatorname{supp}\left(\tau_{0} \rho_{1} \rho_{2} \tau_{3}\right) \subseteq \operatorname{supp}(t), \tau_{0} \rho_{1} \rho_{2} \tau_{3}$ commutes with $t$ and is the product of $r-r_{*}$ pairwise disjoint 3-cycles.

(ii) $\sigma_{1} \sigma_{2} \tau_{0} \sigma_{4}$ commutes with $\tau_{0} \rho_{1} \rho_{2} \tau_{3}$ and is the product of $r-r_{*}$ pairwise disjoint 3 -cycles. Moreover $\operatorname{supp}\left(\sigma_{1} \sigma_{2} \tau_{0} \sigma_{4}\right) \cap \operatorname{supp}(x)=\emptyset$.

(iii) $|\Delta|=s_{*}$ if $n=6 r$ and $|\Delta| \geqslant 1+s_{*}$ if $n \geqslant 6 r$.

Proof. (i) Since $\operatorname{supp}\left(\rho_{1} \rho_{2}\right)=\operatorname{supp}\left(\tau_{1} \tau_{2}\right), \tau_{0} \rho_{1} \rho_{2} \tau_{3}$ is the product of pairwise disjoint 3cycles, and the number of such 3-cycles is $r-r_{*}$. Because $\rho_{1}$ and $\rho_{2}$ both commute with $t, \tau_{0} \rho_{1} \rho_{2} \tau_{3}$ commutes with $t$.

(ii) Since $\operatorname{supp}\left(\sigma_{4}\right) \subseteq \Delta$ and $\operatorname{supp}\left(\tau_{0} \rho_{1} \rho_{2} \tau_{3}\right) \subseteq \operatorname{supp}(t), \sigma_{4}$ commutes with $\tau_{0} \rho_{1} \rho_{2} \tau_{3}$. While $\sigma_{1} \sigma_{2} \tau_{0}$ is a product of 3-cycles which appear in $\tau_{0} \rho_{1} \rho_{2} \tau_{3}$ and therefore $\sigma_{1} \sigma_{2} \tau_{0} \sigma_{4}$ commutes with $\tau_{0} \rho_{1} \rho_{2} \tau_{3}$. By construction $\sigma_{i} \cap \operatorname{supp}(x)=\emptyset(i=1,2), \operatorname{supp}\left(\tau_{0}\right) \cap \operatorname{supp}(x)=\emptyset$ by definition and because we chose $\sigma_{4}$ so as $\operatorname{supp}\left(\sigma_{4}\right) \subseteq \Lambda$ we get $\operatorname{supp}\left(\sigma_{1} \sigma_{2} \tau_{0} \sigma_{4}\right) \cap \operatorname{supp}(x)=$ $\emptyset$.

(iii) Part (iii) follows from $\left|\operatorname{supp}\left(\sigma_{4}\right)\right|=r_{1}+2 r_{2}+3 r_{3}$ and $\Delta=\Lambda \backslash \operatorname{supp}\left(\sigma_{4}\right)$. 
We are now in a position to prove Theorem 1.2.

Proof of Theorem 1.2. Let $y \in X$ be such that $\left|\operatorname{supp}(y) \cap \vartheta_{i}(t)\right|=1=\left|\operatorname{supp}(t) \cap \vartheta_{i}(y)\right|$ for $i=1, \ldots, r$. Then $C_{G}(t) \cap C_{G}(y)=\operatorname{Sym}(\Psi)$ where $\Psi=\Omega \backslash(\operatorname{supp}(t) \cup \operatorname{supp}(y))$. Now $|\operatorname{supp}(t) \cup \operatorname{supp}(y)|=3 r+3 r-r=5 r$ and so $|\Psi|=n-5 r<8 r-5 r=3 r$. Thus $X \cap C_{G}(t) \cap C_{G}(y)=\emptyset$ and consequently $d(t, y) \geqslant 3$. Hence Diam $(\mathcal{C}(G, X)) \geqslant 3$.

Let $x \in X$. We aim to show that $d(t, x) \leqslant 3$. On account of $C_{G}(t)$ having shape $3^{r} \operatorname{Sym}(r) \times \operatorname{Sym}(n-3 r)$ there is no loss in supposing $\tau_{*}=t_{1} \ldots t_{r_{*}}$ where $0 \leqslant r_{*} \leqslant 4$ $\left(r_{*}=0\right.$ meaning $\left.\tau_{*}=1\right)$. Depending on $\tau_{*}$ we define two elements $\rho_{*}$ and $\sigma_{*}$ which will be the product of $r_{*}$ pairwise disjoint 3-cycles.

(1) $r_{*}=4$

Then we have $\tau_{*}=t_{1} t_{2} t_{3} t_{4}=(1,2,3)(4,5,6)(7,8,9)(10,11,12), s_{*}=6$ and we may, without loss, assume $\operatorname{supp}\left(\tau_{*}\right) \cap \operatorname{supp}(x)=\{1,4,7,8,10,11\}$. Observe that $|\operatorname{supp}(x) \backslash \operatorname{supp}(t)| \geqslant$ 6 and so we may select $\alpha_{1}, \alpha_{2}, \alpha_{3}, \alpha_{4}, \alpha_{5}, \alpha_{6} \in \operatorname{supp}(x) \backslash \operatorname{supp}(t)$. Also by Lemma 2.1(iii), as $s_{*}=6,|\Delta| \geqslant 7$. Thus we may also select $\beta_{1}, \beta_{2}, \beta_{3}, \beta_{4}, \beta_{5}, \beta_{6} \in \Delta$. Define

$$
\rho_{*}=\left(\alpha_{1}, \alpha_{2}, \alpha_{3}\right)\left(\alpha_{4}, \alpha_{5}, \alpha_{6}\right)\left(\beta_{1}, \beta_{2}, \beta_{3}\right)\left(\beta_{4}, \beta_{5}, \beta_{6}\right)
$$

and

$$
\sigma_{*}=(2,3,5)(6,9,12)\left(\beta_{1}, \beta_{2}, \beta_{3}\right)\left(\beta_{4}, \beta_{5}, \beta_{6}\right) \text {. }
$$

(2) $r_{*}=3$

So $\tau_{*}=t_{1} t_{2} t_{3}=(1,2,3)(4,5,6)(7,8,9)$. First we examine the case when $s_{*}=4$, and may suppose that $\operatorname{supp}\left(\tau_{*}\right) \cap \operatorname{supp}(x)=\{1,4,7,8\}$. Here we have $|\operatorname{supp}(x) \backslash \operatorname{supp}(t)| \geqslant 5$ and $|\Delta| \geqslant 5$. Choose $\alpha_{1}, \alpha_{2}, \alpha_{3}, \alpha_{4}, \alpha_{5} \in \operatorname{supp}(x) \backslash \operatorname{supp}(t)$ and $\beta_{1}, \beta_{2}, \beta_{3}, \beta_{4}, \beta_{5} \in \Delta$, and define

$$
\rho_{*}=\left(\alpha_{1}, \alpha_{2}, \alpha_{3}\right)\left(\alpha_{4}, \alpha_{5}, \beta_{1}\right)\left(\beta_{2}, \beta_{3}, \beta_{4}\right)
$$

and

$$
\sigma_{*}=(2,3,5)\left(6,9, \beta_{5}\right)\left(\beta_{2}, \beta_{3}, \beta_{4}\right) .
$$

We move onto the case when $s_{*}=5$ and, without loss of generality, assume $\operatorname{supp}\left(\tau_{*}\right) \cap$ $\operatorname{supp}(x)=\{1,2,4,5,7\}$. Since $|\operatorname{supp}(x) \backslash \operatorname{supp}(t)| \geqslant 4$ and $|\Delta| \geqslant 6$, we may select $\alpha_{1}, \alpha_{2}, \alpha_{3} \in \operatorname{supp}(x) \backslash \operatorname{supp}(t)$ and $\beta_{1}, \beta_{2}, \beta_{3}, \beta_{4}, \beta_{5}, \beta_{6} \in \Delta$. Then we take

$$
\rho_{*}=\left(\alpha_{1}, \alpha_{2}, \alpha_{3}\right)\left(\beta_{1}, \beta_{2}, \beta_{3}\right)\left(\beta_{4}, \beta_{5}, \beta_{6}\right)
$$

and

$$
\sigma_{*}=(3,6,8)\left(\beta_{1}, \beta_{2}, \beta_{3}\right)\left(\beta_{4}, \beta_{5}, \beta_{6}\right) .
$$

(3) $r_{*}=2$ 
So $\tau_{*}=t_{1} t_{2}=(1,2,3)(4,5,6)$ with $s_{*}=2,3$ or 4 . First we look at the case when $s_{*}=2$ or 3. Then we have $|\operatorname{supp}(x) \backslash \operatorname{supp}(t)| \geqslant 3$, $|\operatorname{supp}(t) \backslash \operatorname{supp}(x)| \geqslant 3$ and $|\Delta| \geqslant 3$. Choosing $\alpha_{1}, \alpha_{2}, \alpha_{3} \in \operatorname{supp}(x) \backslash \operatorname{supp}(t), \beta_{1}, \beta_{2}, \beta_{3} \in \Delta$ and $\left.\gamma_{1}, \gamma_{2}, \gamma_{3} \in \operatorname{supp}(t) \backslash \operatorname{supp}(x)\right)$, we let

$$
\rho_{*}=\left(\alpha_{1}, \alpha_{2}, \alpha_{3}\right)\left(\beta_{1}, \beta_{2}, \beta_{3}\right)
$$

and

$$
\sigma_{*}=\left(\gamma_{1}, \gamma_{2}, \gamma_{3}\right)\left(\beta_{1}, \beta_{2}, \beta_{3}\right) .
$$

Now assume that $s_{*}=4$, and, without loss, that $\operatorname{supp}\left(\tau_{*}\right) \cap \operatorname{supp}(x)=\{1,2,4,5\}$. Because $|\operatorname{supp}(x) \backslash \operatorname{supp}(t)| \geqslant 2$ and $|\Delta| \geqslant 5$ we may choose $\alpha_{1}, \alpha_{2} \in \operatorname{supp}(x) \backslash \operatorname{supp}(t)$ and $\beta_{1}, \beta_{2}, \beta_{3}, \beta_{4}, \beta_{5} \in \Delta$ and then define

$$
\rho_{*}=\left(\alpha_{1}, \alpha_{2}, \beta_{1}\right)\left(\beta_{2}, \beta_{3}, \beta_{4}\right)
$$

and

$$
\sigma_{*}=\left(3,6, \beta_{5}\right)\left(\beta_{2}, \beta_{3}, \beta_{4}\right)
$$

(4) $r_{*}=1$

Then $\tau_{*}=t_{1}=(1,2,3)$ and $s_{*}=1$ or 2 . Suppose $s_{*}=1$ with $\operatorname{supp}\left(\tau_{*}\right) \cap \operatorname{supp}(x)=\{1\}$. So $|\operatorname{supp}(x) \backslash \operatorname{supp}(t)| \geqslant 2 \leqslant|\Delta|$. Selecting $\alpha_{1}, \alpha_{2} \in \operatorname{supp}(x) \backslash \operatorname{supp}(t)$ and $\beta_{1}, \beta_{2} \in \Delta$, we set

$$
\rho_{*}=\left(\alpha_{1}, \alpha_{2}, \beta_{1}\right)
$$

and

$$
\sigma_{*}=\left(2,3, \beta_{2}\right) .
$$

While if $s_{*}=2$, then $|\Delta| \geqslant 3$ and selecting $\beta_{1}, \beta_{2}, \beta_{3} \in \Delta$ we set

$$
\rho_{*}=\sigma_{*}=\left(\beta_{1}, \beta_{2}, \beta_{3}\right) .
$$

(5) $r_{*}=0$

Here we take $\rho_{*}=1=\sigma_{*}$.

Put $y=\rho_{*} \tau_{0} \rho_{1} \rho_{2} \tau_{3}$. Since $y$ is the product of $r_{*}+r_{0}+r_{1}+r_{2}+r_{3}=r$ disjoint 3cycles, $y \in X$. Further we have that $t y=y t$ by Lemma 2.1(i). Next we consider $z=\sigma_{*} \sigma_{1} \sigma_{2} \tau_{0} \sigma_{4}$. Each of $\sigma_{*} \sigma_{1}, \sigma_{2}, \tau_{0}$ and $\sigma_{4}$ are pairwise disjoint. Recalling that $\sigma_{1}, \sigma_{2}$ and $\sigma_{4}$ are, respectively, the product of $\frac{2 r_{1}}{3}, \frac{r_{2}}{3},\left(\frac{r_{1}}{3}+\frac{2 r_{2}}{3}+r_{3}\right)$ disjoint 3-cycles, we see that $z \in X$. It may be further checked using Lemma 2.1(ii) that $y z=z y$ and $x z=z x$, and consequently $d(t, x) \leqslant 3$. This completes the proof of Theorem 1.2 .

Proof of Theorem 1.3. Let $x \in X$. Our objective here is to show that $d(t, x) \leqslant 4$ from which it will follow that $\operatorname{Diam}(\mathcal{C}(G, X)) \leqslant 4$. We proceed in a similar fashion to that in the proof of Theorem 1.1 though here, except for some cases, we will define three permutations $\rho_{*}, \sigma_{*}, \xi_{*}$, each a product of $r_{*}$ pairwise disjoint cycles. 
(6) $r_{*}=4$

So $\tau_{*}=t_{1} t_{2} t_{3} t_{4}=(1,2,3)(4,5,6)(7,8,9)(10,11,12)$ with $s_{*}=6$. Assume, without loss, that $\operatorname{supp}\left(\tau_{*}\right) \cap \operatorname{supp}(x)=\{1,4,7,8,10,11\}$. Since $|\operatorname{supp}(x) \backslash \operatorname{supp}(t)| \geqslant 6$ and so we may choose $\alpha_{1}, \alpha_{2}, \alpha_{3}, \alpha_{4}, \alpha_{5}, \alpha_{6} \in \operatorname{supp}(x) \backslash \operatorname{supp}(t)$. Further, as $|\Delta|=s_{*}=6$ by Lemma 2.1(iii), we may also choose $\beta_{1}, \beta_{2}, \beta_{3}, \beta_{4}, \beta_{5}, \beta_{6} \in \Delta$. Now define

$$
\rho_{*}=\left(\alpha_{1}, \alpha_{2}, \alpha_{3}\right)\left(\alpha_{4}, \alpha_{5}, \alpha_{6}\right)\left(\beta_{1}, \beta_{2}, \beta_{3}\right)\left(\beta_{4}, \beta_{5}, \beta_{6}\right)
$$

and

$$
\sigma_{*}=(2,3,5)(6,9,12)\left(\beta_{1}, \beta_{2}, \beta_{3}\right)\left(\beta_{4}, \beta_{5}, \beta_{6}\right)
$$

(7) $r_{*}=3$

So $\tau_{*}=t_{1} t_{2} t_{3}=(1,2,3)(4,5,6)(7,8,9)$. If $s_{*}=4$ we may suppose without loss that $\operatorname{supp}\left(\tau_{*}\right) \cap \operatorname{supp}(x)=\{1,4,7,8\}$. Here we have $|\operatorname{supp}(x) \backslash \operatorname{supp}(t)| \geqslant 5$ and $|\Delta|=s_{*}=4$ by Lemma 2.1(iii). Choose $\alpha_{1}, \alpha_{2}, \alpha_{3} \in \operatorname{supp}(x) \backslash \operatorname{supp}(t)$ and $\beta_{1}, \beta_{2}, \beta_{3} \in \Delta$, and define

$$
\begin{gathered}
\rho_{*}=\left(\alpha_{1}, \alpha_{2}, \alpha_{3}\right)\left(\beta_{1}, \beta_{2}, \beta_{3}\right)(1,2,3), \\
\sigma_{*}=(5,6,9)\left(\beta_{1}, \beta_{2}, \beta_{3}\right)(1,2,3)
\end{gathered}
$$

and

$$
\xi_{*}=(5,6,9)\left(\beta_{1}, \beta_{2}, \beta_{3}\right)(\alpha, \beta, \gamma),
$$

where $(\alpha, \beta, \gamma)$ is a 3-cycle of $x$ for which $1 \notin\{\alpha, \beta, \gamma\}$. Note that $\{\alpha, \beta, \gamma\} \cap \operatorname{supp}\left(\sigma_{*}\right)=\emptyset$. For the case when $s_{*}=5$, without loss of generality, we assume $\operatorname{supp}\left(\tau_{*}\right) \cap \operatorname{supp}(x)=$ $\{1,4,5,7,8\}$. Since $|\operatorname{supp}(x) \backslash \operatorname{supp}(t)| \geqslant 4$ and $|\Delta|=s_{*}=5$, we may select $\alpha_{1}, \alpha_{2}, \alpha_{3} \in$ $\operatorname{supp}(x) \backslash \operatorname{supp}(t)$ and $\beta_{1}, \beta_{2}, \beta_{3} \in \Delta$. Then we take

$$
\begin{gathered}
\rho_{*}=\left(\alpha_{1}, \alpha_{2}, \alpha_{3}\right)\left(\beta_{1}, \beta_{2}, \beta_{3}\right)(4,5,6), \\
\sigma_{*}=(2,3,9)\left(\beta_{1}, \beta_{2}, \beta_{3}\right)(4,5,6)
\end{gathered}
$$

and

$$
\xi_{*}=(2,3,9)\left(\beta_{1}, \beta_{2}, \beta_{3}\right)(\alpha, \beta, \gamma),
$$

where $(\alpha, \beta, \gamma)$ is a 3 -cycle of $x$ chosen so as $\{4,5\} \cap\{\alpha, \beta, \gamma\}=\emptyset$. Since $r \geqslant r_{*}=3$ such a choice is possible.

Before dealing with $r_{*}=2$ we analyze a number of small cases.

(8) Suppose that $t=(1,2,3)(4,5,6)$ (so $r=2$ and $n=12$ ).

(i) If $x=(1,7,8)(4,9,10)$ or $x=(1,4,7)(2,5,8)$, then $d(t, x) \leqslant 4$.

(ii)If $x=(1,4,7)(8,9,10)$, then $d(t, x) \leqslant 3$. 
Assume that $x=(1,7,8)(4,9,10)$, and let

$$
x_{1}=(7,8,11)(9,10,12), \quad x_{2}=(2,3,5)(9,10,12), \quad x_{3}=(2,3,5)(1,7,8) .
$$

Then $x_{1}, x_{2}, x_{3} \in X$ and $\left(t, x_{1}, x_{2}, x_{3}, x\right)$ is a path in $\mathcal{C}(G, X)$ whence $d(t, x) \leqslant 4$. In the case $x=(1,4,7)(2,5,8)$ we take $x_{1}=(7,8,9)(10,11,12), x_{2}=(1,3,6)(10,11,12)$ and $x_{3}=(2,5,8)(10,11,12)$. It is easily checked that $\left(t, x_{1}, x_{2}, x_{3}, x\right)$ is also a path in $\mathcal{C}(G, X)$, so proving part (i). For $x=(1,4,7)(8,9,10)$ taking $x_{1}=(1,2,3)(8,9,10)$ and $x_{2}=(5,6,11)(8,9,10)$ gives a path $\left(t, x_{1}, x_{2}, x\right)$ in $\mathcal{C}(G, X)$. So (ii) holds and (8) is proved.

(9) Suppose $t=(1,2,3)(4,5,6)(7,8,9)$ with $\tau_{*}=(1,2,3)(4,5,6)$ (so $r=3$ and $\left.n=18\right)$. Let $x \in X$ be such that $\operatorname{supp}\left(\tau_{*}\right) \cap \operatorname{supp}(x)=\{1,4\}$ and assume 1 and 4 are in different 3 -cycles of $x$. Then $d(t, x) \leqslant 4$.

By assumption $x=(1, *, *)(4, \delta, \epsilon)(\alpha, \beta, \gamma)$ with $\{1,4\} \cap\{\alpha, \beta, \gamma\}=\emptyset$. Because $\tau_{*}=$ $(1,2,3)(4,5,6)$ we must have $\operatorname{supp}(t) \cap \operatorname{supp}(x)=\{1,4\}$ or $\{1,4,7,8,9\}$. Suppose the former holds and set $x_{1}=(1,2,3)(\alpha, \beta, \gamma)(7,8,9)$ and $x_{2}=(4, \delta, \epsilon)(\alpha, \beta, \gamma)(7,8,9)$. Then $\left(t, x_{1}, x_{2}, x\right)$ is a path in $\mathcal{C}(G, X)$. Hence $d(t, x) \leqslant 3$. Turning to the latter case we have $|\operatorname{supp}(t) \cup \operatorname{supp}(x)|=13$. So we may choose, say, $16,17,18 \in \Lambda$ and then take $x_{1}=(1,2,3)(4,5,6)(16,17,18), x_{2}=(1,2,3)(\alpha, \beta, \gamma)(16,17,18)$ and $x_{3}=$ $(4, \delta, \epsilon)(\alpha, \beta, \gamma)(16,17,18)$, giving a path $\left(t, x_{1}, x_{2}, x_{3}, x\right)$ in $\mathcal{C}(G, X)$. Thus $d(t, x) \leqslant 4$, so proving (9).

(10) $r_{*}=2$

So we have $\tau_{*}=t_{1} t_{2}=(1,2,3)(4,5,6)$ with $s_{*}=2,3$ or 4 . First we consider the case $s_{*}=2$, and assume $\operatorname{supp}\left(\tau_{*}\right) \cap \operatorname{supp}(x)=\{1,4\}$. For the moment also assume that $r=2$ (so $t=\tau_{*}$ ). Then, without loss, $x$ is either $(1,7,8)(4,9,10)$ (1 and 4 in different 3 -cycles of $x$ ) or $(1,4,7)(8,9,10)$ (1 and 4 in the same 3 -cycle of $x$ ). By (8)(i) we have $d(t, x) \leqslant 4$. So, since we are aiming to show that $d(t, x) \leqslant 4$, we may suppose $r \geqslant 3$. Now consider the possibility that $r=3$ and 1 and 4 are in different 3-cycles of $x$. Then, without loss, $x=(1, *, *)(4, \delta, \epsilon)(\alpha, \beta, \gamma)$ in which case $d(t, x) \leqslant 4$ by $(9)$. Thus, when $r=3$, we may suppose 1 and 4 are in the same 3-cycle of $x$. Consequently, as $r \geqslant 3$, we may find two 3 -cycles of $x,(\alpha, \beta, \gamma)$ and $(\delta, \epsilon, \lambda)$ such that $\{\alpha, \beta, \gamma, \delta, \epsilon, \lambda\} \cap\{1,4\}=\emptyset$. Now we define $\rho_{*}, \sigma_{*}$ and $\xi_{*}$ by taking $\rho_{*}=\sigma_{*}=\tau_{*}$ and $\xi_{*}=(\alpha, \beta, \gamma),(\delta, \epsilon, \lambda)$.

Next we look at the case $s_{*}=3$. Then we have $|\operatorname{supp}(x) \backslash \operatorname{supp}(t)| \geqslant 3,|\operatorname{supp}(t) \backslash \operatorname{supp}(x)| \geqslant$ 3 and $|\Delta|=s_{*}=3$. Choosing $\alpha_{1}, \alpha_{2}, \alpha_{3} \in \operatorname{supp}(x) \backslash \operatorname{supp}(t), \beta_{1}, \beta_{2}, \beta_{3} \in \Delta$ and $\gamma_{1}, \gamma_{2}, \gamma_{3} \in$ $\operatorname{supp}(t) \backslash \operatorname{supp}(x))$, we let

$$
\rho_{*}=\left(\alpha_{1}, \alpha_{2}, \alpha_{3}\right)\left(\beta_{1}, \beta_{2}, \beta_{3}\right)
$$

and

$$
\sigma_{*}=\left(\gamma_{1}, \gamma_{2}, \gamma_{3}\right)\left(\beta_{1}, \beta_{2}, \beta_{3}\right) \text {. }
$$

Finally we come to $s_{*}=4$. So without loss we have $\operatorname{supp}\left(\tau_{*}\right) \cap \operatorname{supp}(x)=\{1,2,4,5\}$. Suppose, for the moment, that for all 3-cycles $(\alpha, \beta, \gamma)$ we have $\{1,2\} \cap\{\alpha, \beta, \gamma\} \neq \emptyset \neq$ 
$\{4,5\} \cap\{\alpha, \beta, \gamma\}$. Then it follows that $r=2$ and, without loss, $x=(1,4,7)(2,5,8)$. But then $d(t, x) \leqslant 4$ by (8)(ii). Thus we may suppose $x$ contains a 3 -cycle $(\alpha, \beta, \gamma)$ such that $(\alpha, \beta, \gamma) \cap\{1,2\}=\emptyset$, and we can now define $\rho_{*}$ and $\sigma_{*}$. Since $|\Delta|=s_{*}=4$, we have $\beta_{1}, \beta_{2}, \beta_{3} \in \Delta$. Let $\rho_{*}=(1,2,3)\left(\beta_{1}, \beta_{2}, \beta_{3}\right)$ and $\sigma_{*}=(\alpha, \beta, \gamma)\left(\beta_{1}, \beta_{2}, \beta_{3}\right)$. This completes the case $s_{*}=4$ and $(10)$.

Yet another special case must be looked at before doing $r_{*}=1$.

(11) Let $t=(1,2,3)(4,5,6)$ with $\tau_{*}=(1,2,3)$. Suppose $x=(1, *, *)(2, *, *) \in X$ with $\operatorname{supp}\left(\tau_{*}\right) \cap \operatorname{supp}(x)=\{1,2\}$. Then $d(t, x) \leqslant 3$.

Since $\tau_{*}=(1,2,3), \operatorname{supp}(t) \cap \operatorname{supp}(x)=\{1,2\}$ or $\{1,2,4,5,6\}$. If $\operatorname{supp}(t) \cap \operatorname{supp}(x)=\{1,2\}$ and, say $\Omega \backslash(\operatorname{supp}(t) \cap \operatorname{supp}(x))=\{11,12\}$, then define $x_{1}=(4,5,6)(10,11,12), x_{2}=$ $(4,5,6)(\alpha, \beta, \gamma)$ where $(\alpha, \beta, \gamma)$ is a 3 -cycle not containing 10 . While in the other case with, say $\Omega \backslash(\operatorname{supp}(t) \cap \operatorname{supp}(x))=\{8,9,10,11,12\}$ we define $x_{1}=(8,9,10)(7,11,12), x_{2}=$ $(8,9,10)(\alpha, \beta, \gamma)$ where $(\alpha, \beta, \gamma)$ is a 3 -cycle not containing 7 . Hence $d(t, x) \leqslant 3$.

(12) $r_{*}=1$

So we have either, without loss, $\operatorname{supp}\left(\tau_{*}\right) \cap \operatorname{supp}(x)=\{1\}$ or $\{2,3\}$. In view of (10), as $r>1$, either $d(t, x) \leqslant 3$ or we may find a 3-cycle $(\alpha, \beta, \gamma)$ of $x$ for which $\operatorname{supp}\left(\tau_{*}\right) \cap$ $\{\alpha, \beta, \gamma\}=\emptyset$. In the latter case we define $\rho_{*}=\sigma_{*}=\tau_{*}$ and $\xi_{*}=(\alpha, \beta, \gamma)$.

(13) $r_{*}=0$

Just as in (5) we take $\rho_{*}=1=\sigma_{*}$.

Now let $y=\rho_{*} \tau_{0} \rho_{1} \rho_{2} \tau_{3}, z=\sigma_{*} \sigma_{1} \sigma_{2} \tau_{0} \sigma_{4}$ and $w=\xi_{*} \sigma_{1} \sigma_{2} \tau_{0} \sigma_{4}$ (where $w$ is only defined if in (6), (7), (10), (12), (13) $\xi_{*}$ is defined). Then $y, z, w \in X$ with $(t, y, z, w, x)$ is a path in $\mathcal{C}(G, X)$. Consequently $d(t, x) \leqslant 4$. Since $x$ was an arbitrary vertex, this shows that Diam $(\mathcal{C}(G, X)) \leqslant 4$ and completes the proof of Theorem 1.3.

We end this paper with a table containing some calculations on diameters and discs using Magma[10]. Each entry in the table first gives the size of the relevant $\Delta_{i}(t)$ for the given $r$ and $n$ with the number in brackets being the number of $C_{G}(t)$-orbits on $\Delta_{i}(t)$. A blank entry means that $\left|\Delta_{i}(t)\right|=0$. 


\begin{tabular}{|c|c|c|c|c|c|c|}
\hline & $\Delta_{1}(t)$ & $\Delta_{2}(t)$ & $\Delta_{3}(t)$ & $\Delta_{4}(t)$ & $\Delta_{5}(t)$ & $\Delta_{6}(t)$ \\
\hline $\begin{array}{l}r=1 \\
n=7 \\
n=8 \\
n=9\end{array}$ & $\begin{array}{c}9(2) \\
21(2) \\
41(2)\end{array}$ & $\begin{array}{c}24(2) \\
90(3) \\
126(3)\end{array}$ & $\begin{array}{c}36(1) \\
- \\
-\end{array}$ & $\begin{array}{l}- \\
- \\
-\end{array}$ & $\begin{array}{l}- \\
- \\
-\end{array}$ & $\begin{array}{l}- \\
- \\
-\end{array}$ \\
\hline $\begin{array}{l}\boldsymbol{r}=\mathscr{2} \\
n=10 \\
n=11 \\
n=12 \\
n=13 \\
n=14 \\
n=15 \\
n=16\end{array}$ & $\begin{array}{c}35(4) \\
83(4) \\
203(5) \\
563(5) \\
1,571(5) \\
4,035(5) \\
9,363(5)\end{array}$ & $\begin{array}{c}192(6) \\
1,080(9) \\
6,300(16) \\
25,740(30) \\
67,140(48) \\
168,948(54) \\
310,956(55)\end{array}$ & $\begin{array}{c}1,008(10) \\
7,560(23) \\
28,296(34) \\
42,336(25) \\
51,408(7) \\
27,216(1) \\
- \\
\end{array}$ & $\begin{array}{c}2,628(20) \\
9,756(23) \\
2,160(5) \\
- \\
- \\
- \\
- \\
-\end{array}$ & $\begin{array}{c}3,672(13) \\
- \\
- \\
- \\
- \\
- \\
-\end{array}$ & $\begin{array}{c}864(5) \\
- \\
- \\
- \\
- \\
- \\
- \\
\end{array}$ \\
\hline $\begin{array}{l}r=3 \\
n=9 \\
n=12 \\
n=13 \\
n=14 \\
n=15 \\
n=16 \\
n=17 \\
n=18\end{array}$ & $\begin{array}{c}25(4) \\
49(7) \\
121(7) \\
265(7) \\
745(9) \\
2,545(9) \\
8,089(9) \\
24,441(10)\end{array}$ & $\begin{array}{c}216(4) \\
648(8) \\
2,808(18) \\
9,936(23) \\
62,424(46) \\
482,760(90) \\
3,400,272(145) \\
16,126,398(210)\end{array}$ & $\begin{array}{c}1,512(11) \\
9,936(39) \\
79,488(85) \\
390,582(138) \\
2,414,610(243) \\
17,798,778(578) \\
50,175,126(728) \\
92,757,960(679)\end{array}$ & $\begin{array}{c}486(6) \\
90,990(139) \\
724,086(383) \\
3,217,806(564) \\
8,733,420(594) \\
7,341,516(220) \\
870,912(16) \\
- \\
\end{array}$ & $\begin{array}{c}- \\
327,024(404) \\
783,432(332) \\
865,890(143) \\
- \\
- \\
- \\
-\end{array}$ & $\begin{array}{c}- \\
64,152(102) \\
11,664(3) \\
- \\
- \\
- \\
- \\
-\end{array}$ \\
\hline
\end{tabular}

Continued on Next Page... 
Table 1 - Continued

\begin{tabular}{|l|c|c|c|c|c|c|}
\hline & $\Delta_{1}(t)$ & $\Delta_{2}(t)$ & $\Delta_{3}(t)$ & $\Delta_{4}(t)$ & $\Delta_{5}(t)$ & $\Delta_{6}(t)$ \\
\hline $\boldsymbol{r}=\mathbf{4}$ & & & & & & \\
$n=12$ & $159(6)$ & $8,532(20)$ & $193,104(121)$ & $44,604(37)$ & - & \\
$n=15$ & $367(11)$ & $37,044(52)$ & $3,053,160(682)$ & $81,668,484(8,294)$ & & - \\
$n=16$ & $991(11)$ & $271,236(92)$ & $56,926,656(2,351)$ & $390,829,212(13,122)$ & $419,904(12)$ & - \\
$n=17$ & $2,239(11)$ & $1,350,612(112)$ & $487,124,064(4,539)$ & $1,036,246,284(12,578)$ & - & - \\
\hline \hline $\boldsymbol{r}=\mathbf{5}$ & & & & & & - \\
$n=15$ & $751(8)$ & $154,440(44)$ & $17,669,304(783)$ & $27,020,304(996)$ & - & - \\
\hline
\end{tabular}

Table 1: Disc sizes and $C_{G}(t)$-orbits 


\section{References}

[1] Aschbacher, M.; Segev, Y., The uniqueness of groups of Lyons type. J. Amer. Math. Soc. 5 (1992), no. 1, 75-98.

[2] Bates, C.; Bundy, D.; Perkins, S.; Rowley, P. Commuting involution graphs for symmetric groups. J. Algebra 266 (2003), no. 1, 133-153.

[3] Bates, C.; Bundy, D.; Perkins, S.; Rowley, P. Commuting involution graphs for finite Coxeter groups. J. Group Theory 6 (2003), no. 4, 461-476.

[4] Bates, C.; Bundy, D.; Perkins, S.; Rowley, P. Commuting involution graphs in special linear groups. Comm. Algebra 32 (2004), no. 11, 4179-4196.

[5] Bates, C.; Bundy, D.; Hart, S.; Rowley, P. Commuting involution graphs for sporadic simple groups. J. Algebra 316 (2007), no. 2, 849-868.

[6] Bates, C.; Bundy, D.; Hart, S.; Rowley, P. A Note on Commuting Graphs for Symmetric Groups, Electron. J. Combin. 16(1) (2009), \#R6.

[7] Baumeister, B.; Stein, A. Commuting graphs of odd prime order elements in simple groups. arXiv:0908.2583

[8] Brauer, R.; Fowler, K. A., On groups of even order. Ann. Math. (2) 62 (1955), 565583.

[9] Bundy, D. The connectivity of commuting graphs. J. Combin. Theory Ser. A 113 (2006), no. 6, 995-1007.

[10] Cannon, J.J; Playoust, C. An Introduction to Algebraic Programming with MAgma, Springer-Verlag (1997).

[11] Everett, A., Commuting involution graphs for 3-dimensional unitary groups. Electron. J. Combin. 18(1) (2011),\#P103,

[12] Everett, A.; Rowley, P., Commuting Involution Graphs for 4Dimensional Projective Symplectic Groups. Preprint, 2010. Available from http://eprints.ma.man.ac.uk/1564/

[13] Fischer, B., Finite groups generated by 3-transpositions. I. Invent. Math. 13 (1971), 232-246.

[14] Segev, Y., On finite homomorphic images of the multiplicative group of a division algebra. Ann. Math. (2) 149 (1999), no. 1, 219-251.

[15] Segev, Y., The commuting graph of minimal nonsolvable groups. Geom. Dedicata 88 (2001), no. 1-3, 55-66.

[16] Segev, Y.; Seitz, G.M. Anisotropic groups of type $A_{n}$ and the commuting graph of finite simple groups. Pacific J. Math. 202 (2002), no. 1, 125-225. 